PROCEEDINGS OF THE

AMERICAN MATHEMATICAL SOCIETY

Volume 134, Number 11, November 2006, Pages 3107-3114

S 0002-9939(06)08385-7

Article electronically published on May 11, 2006

\title{
NONABELIAN FREE SUBGROUPS IN HOMOMORPHIC IMAGES OF VALUED QUATERNION DIVISION ALGEBRAS
}

\author{
ANDREI S. RAPINCHUK, LOUIS ROWEN, AND YOAV SEGEV
}

(Communicated by Jonathan I. Hall)

\begin{abstract}
Given a quaternion division algebra $D$, a noncentral element $e \in$ $D^{\times}$is called pure if its square belongs to the center. A theorem of Rowen and Segev (2004) asserts that for any quaternion division algebra $D$ of positive characteristic $>2$ and any pure element $e \in D^{\times}$the quotient $D^{\times} / X(e)$ of $D^{\times}$by the normal subgroup $X(e)$ generated by $e$, is abelian-by-nilpotent-by-abelian. In this note we construct a quaternion division algebra $D$ of characteristic zero containing a pure element $e \in D$ such that $D^{\times} / X(e)$ contains a nonabelian free group. This demonstrates that the situation in characteristic zero is very different.
\end{abstract}

\section{INTRODUCTION}

Let $D$ be a quaternion division algebra with center $K$. An element $e \in D \backslash K$ is called pure if $e^{2} \in K$. Given an element $a \in D \backslash K$, we denote by

$$
X(a) \text { and } Y(a)
$$

the normal subgroups of $D^{\times}$generated by $\langle a\rangle$ and $S L_{1}(K(a))$ respectively, where as usual $S L_{1}(K(a))=K(a) \cap S L_{1}(D)$ and $S L_{1}(D)$ is the subgroup of elements having reduced norm 1.

In [7] quotients of the form $D^{\times} / X(e)$ were considered for pure elements $e \in$ $D^{\times}$. These quotients arise in the analysis of the Whitehead group $W(G, k)$ of an absolutely simple simply connected algebraic $k$-group $G$ of type ${ }^{3,6} D_{4}$ having $k$ rank 1 (see Tits's Bourbaki talk [1] for the relevant terminology). It was shown that if $D$ has positive characteristic $>2$, then $D^{\times} / X(e)$ is abelian-by-nilpotent-byabelian for any pure element $e \in D^{\times}$, which, by the explicit description of $W(G, k)$ given in [5], implies the solvability of $W(G, k)$ for $G$ as above over a field $k$ of characteristic $>2$. Even though the solvability of $W(G, k)$ is expected to hold in any characteristic (see [6] for a general conjecture), the results of this note indicate

Received by the editors March 3, 2005 and, in revised form, May 14, 2005.

2000 Mathematics Subject Classification. Primary 16K20, 16U60; Secondary 20G15, 12J20.

Key words and phrases. Quaternion division algebra, multiplicative group, valuation, residue algebra.

The first author was partially supported by BSF grant 2000-171, and by NSF grants DMS0138315 and DMS-0502120.

The second author was partially supported by the Israel Science Foundation Center of Excellence.

The third author was partially supported by BSF grant 2000-171.

(C)2006 American Mathematical Society 
that a more delicate analysis will be needed to prove this in characteristic zero. More precisely, we will prove the following.

Theorem 1.1. There exists a quaternion division algebra $D$ of characteristic zero and pure quaternions $e, f \in D$ such that the quotients $D^{\times} / X(e)$ and $D^{\times} / Y(f)$ contain nonabelian free groups. (In particular these quotients are nonsolvable.)

In $₫ 3$ we construct a quaternion division algebra $D$ that supports a valuation $v$ such that the residue algebra $\bar{D}_{v}$ is non-commutative of characteristic two over the residue field $\bar{K}_{v}$. This algebra $D$ has the properties asserted in Theorem 1.1 by the following more general result.

Theorem 1.2. Let $D$ be a quaternion division algebra of characteristic zero that supports a valuation $v$ for which the residue algebra $\bar{D}_{v}$ is non-commutative and has characteristic two. Then $D$ contains pure quaternions $e$ and $f$ such that the quotients $D^{\times} / X(e)$ and $D^{\times} / Y(f)$ have homomorphic images containing $\bar{D}_{v}^{\times} / \bar{K}_{v}^{\times}$, and consequently $D^{\times} / X(e)$ and $D^{\times} / Y(f)$ contain nonabelian free groups.

The proof of Theorem 1.2 proceeds as follows. Let $D$ be a quaternion division algebra as in Theorem 1.2 and let $e, f \in D$ be pure quaternions such that $e f=$ $-f e$. We first observe that $Y(e)$ commutes both with $X(e)$ and $Y(f)$ modulo the congruence subgroup $1+\mathfrak{m}_{D, v}$, where $\mathfrak{m}_{D, v}$ is the valuation ideal. (This follows from a stronger result that comes from [7; see Lemma 2.1 for a short proof). We then apply the following lemma which is an easy consequence of the Cartan-Brauer-Hua Theorem (see, e.g., [3, Theorem 3.9.2, pg. 144]).

Lemma 1.3. Let $D$ be a finite-dimensional division algebra with center $K$ that supports a valuation $v$ so that the residue division algebra $\bar{D}_{v}$ is not commutative. Let $\mathcal{U}_{v}:=\left\{x \in D^{\times} \mid v(x)=0\right\}$ be the group of units and $\mathfrak{m}_{D, v}:=\left\{x \in D^{\times} \mid\right.$ $v(x)>0\}$ be the valuation ideal. Let $*: D^{\times} \rightarrow D^{\times} / K^{\times}\left(1+\mathfrak{m}_{D, v}\right)$ be the canonical homomorphism. Suppose $N, M$ are normal subgroups of $D^{\times}$such that $[N, M]^{*}=$ $1^{*}$. Then

(1) $\mathcal{U}_{v}^{*}=\bar{D}_{v}^{\times} / \bar{K}_{v}^{\times}$, and either $\left(\left(M K^{\times}\right) \cap \mathcal{U}_{v}\right)^{*}=1^{*}$ or $\left(\left(N K^{\times}\right) \cap \mathcal{U}_{v}\right)^{*}=1^{*}$;

(2) for $H \in\{M, N\}$ such that $\left(\left(H K^{\times}\right) \cap \mathcal{U}_{v}\right)^{*}=1^{*}$ we have

$$
\left(H K^{\times} \mathcal{U}_{v}\right) /\left(H K^{\times}\left(1+\mathfrak{m}_{D, v}\right)\right) \cong \bar{D}_{v}^{\times} / \bar{K}_{v}^{\times} .
$$

In $\$ 3$ we extend a well-known construction of valuations on fields of rational functions (cf. 2, Section 10.1, Proposition 2]) to finite-dimensional division algebras of non-commutative rational functions. We then apply this construction to obtain the quaternion division algebra $D$ satisfying the hypotheses of Theorem 1.2 (see Proposition 3.4 and Corollary 3.5). The center of the resulting algebra $D$ has transcendence degree 2 over $\mathbb{Q}$, so in this context we would like to mention that for a finite-dimensional division algebra $\mathcal{D}$ over a global field, any quotient of $S L_{1}(\mathcal{D})$ by a noncentral subgroup is finite and solvable (see [6]), implying that all quotients of $\mathcal{D}^{\times}$by a noncentral subgroup are solvable.

In a preliminary version of this paper (see 9] for a report on this joint result) we proved a weaker version of Theorem 1.1 using ultra-products, however now we have a stronger result that does not require the use of ultra-products. Also, since our construction is explicit it is possible that the algebra $D$ we construct could be used to demonstrate further properties of the Whitehead group. 


\section{The Proof of Theorem 1.2} [7.

In this section we prove Theorem 1.2. We start with a lemma that comes from

Lemma 2.1. Let $D$ be a quaternion division algebra of characteristic $\neq 2$, let $S$ be the (normal) subgroup of $D^{\times}$generated by the set $1+2 S L_{1}(D)$, and let $\bullet: D^{\times} \rightarrow$ $D^{\bullet}:=D^{\times} / S$ be the canonical homomorphism. Then

(1) for any pure $e \in D$, we have $[Y(e), X(e)]^{\bullet}=1^{\bullet}$;

(2) for any pure e, $f \in D$ such that $e f=-f e$, we have $[Y(e), Y(f)]^{\bullet}=1^{\bullet}$.

Proof. First observe that

$$
\left[x^{\bullet},(x+s)^{\bullet}\right]=1^{\bullet}, \quad \text { for all } x \in D^{\times} \text {and } s \in S \text { such that } x+s \neq 0,
$$

because $(x+s)^{\bullet}=\left(s\left(s^{-1} x+1\right)\right)^{\bullet}=\left(s^{-1} x+1\right)^{\bullet}$, and $\left(s^{-1} x+1\right)^{\bullet}$ commutes with $\left(s^{-1} x\right)^{\bullet}=x^{\bullet}$. Next, using the notation $[a, b]=a^{-1} b^{-1} a b, a^{b}=b^{-1} a b$,

$$
\left[g,(1+x)^{-1}\right]=\left(1+x^{g}\right)(1+x)^{-1} \quad \forall x, g \in D^{\times} \text {with } x \neq-1,
$$

and hence

$$
\begin{gathered}
{\left[x^{g}, \frac{1-x}{1+x}\right]^{\bullet}=\left[x^{g}, 1-2(1+x)^{-1}\right]^{\bullet}=\left[x^{g},\left(1+x^{g}\right)^{-1}\left(1+x^{g}-2\left[g,(1+x)^{-1}\right]\right)\right]^{\bullet}} \\
=\left[x^{g}, x^{g}+\left(1-2\left[g,(1+x)^{-1}\right]\right)\right]^{\bullet}=1^{\bullet},
\end{gathered}
$$

where the last equality follows from (1i) using the fact that $1-2\left[g,(1+x)^{-1}\right] \in S$. Replacing $x$ by $\alpha e$, where $\alpha \in K^{\times}$is arbitrary and observing that any element $\neq-1$ in $\mathrm{SL}_{1}(K(e))$ has the form $\frac{1-\alpha e}{1+\alpha e}$ (for example, by Hilbert's Theorem 90), we get (1).

Part (2) follows from (1) because

$$
[e, 1+\alpha f]=\left((1+\alpha f)^{e}\right)^{-1}(1+\alpha f)=(1-\alpha f)^{-1}(1+\alpha f), \text { for all } \alpha \in K^{\times},
$$

and as we mentioned above any element $\neq-1$ of $\mathrm{SL}_{1}(K(f))$ has the form $(1-\alpha f)^{-1}(1+\alpha f)$ for some $\alpha \in K^{\times}$. Hence $\mathrm{SL}_{1}(K(f)) \leq X(e)$, and so $(2)$ follows from (1).

The next lemma is a consequence of the Cartan-Brauer-Hua theorem and will be applied in the proof of Lemma 1.3

Lemma 2.2. Let $D$ be a finite-dimensional division algebra with center $K$. Let $A$ and $B$ be two normal subgroup of $D^{\times}$such that $[A, B] \leq K^{\times}$. Then either $A \subseteq K^{\times}$ or $B \subseteq K^{\times}$.

Proof. First observe that

$$
\text { if }[A, B]=1 \text {, then either } A \subseteq K \text { or } B \subseteq K .
$$

To prove (iii) note that the $K$-subalgebra $K[A]$ generated by $A$ is a division subalgebra normalized by $D^{\times}$, so by the Cartan-Brauer-Hua Theorem (see 3 , Theorem 3.9 .2 , pg. 144] for an easy proof), if $A$ is noncentral, then $K[A]=D$, and since $B$ centralizes $K[A]$ it follows that $B$ is central.

Assume now that $B$ is noncentral. Since $[B, A, A]=[A, B, A]=1$, the three subgroup lemma ([1, (8.7)]) implies that $[[A, A], B]=1$. Since $B$ is noncentral, it follows from (iii) that $[A, A] \leq K^{\times}$. But this shows that $A$ is nilpotent. Hence $A \leq K^{\times}$since by a theorem of Scott $\left[8, D^{\times}\right.$contains no noncentral normal solvable subgroups. 
Proof of Lemma 1.3. (1) Let $\mathcal{O}_{D, v}:=\left\{x \in D^{\times} \mid v(x) \geq 0\right\} \cup\{0\}$ be the valuation ring of $v$. Of course the canonical homomorphism $\mathcal{O}_{D, v} \rightarrow \bar{D}_{v}$ restricted to $\mathcal{U}_{v}$ induces a surjective group homomorphism $\mathcal{U}_{v} \rightarrow \bar{D}_{v}^{\times}$, with kernel $1+\mathfrak{m}_{D, v}$, so $\mathcal{U}_{v} /\left(1+\mathfrak{m}_{D, v}\right)=\bar{D}_{v}^{\times}$and therefore $\mathcal{U}_{v}^{*} \cong \bar{D}_{v}^{\times} / \bar{K}_{v}^{\times}$.

Set $A:=\left(M K^{\times}\right) \cap \mathcal{U}_{v}$ and $B:=\left(N K^{\times}\right) \cap \mathcal{U}_{v}$. Let $^{-}: \mathcal{U}_{v} \rightarrow \mathcal{U}_{v} /\left(1+\mathfrak{m}_{D, v}\right)=\bar{D}_{v}^{\times}$ be the canonical homomorphism. Then $[\bar{A}, \bar{B}] \leq \bar{K}_{v}$. Since $\bar{D}_{v}$ is not commutative Lemma 2.2 implies that either $\bar{A} \leq \bar{K}_{v}$ or $\bar{B} \leq \bar{K}_{v}$, that is, either $A^{*}=1^{*}$ or $B^{*}=1^{*}$.

(2) Suppose $A^{*}=1^{*}$, that is, $\left(M K^{\times}\right) \cap \mathcal{U}_{v} \leq K^{\times}\left(1+\mathfrak{m}_{D, v}\right)$. Then

$$
\begin{gathered}
\left(M K^{\times} \mathcal{U}_{v}\right) /\left(M K^{\times}\left(1+\mathfrak{m}_{D, v}\right)\right) \cong \mathcal{U}_{v} / \mathcal{U}_{v} \cap\left(M K^{\times}\left(1+\mathfrak{m}_{D, v}\right)\right) \\
\cong \mathcal{U}_{v} /\left(\mathcal{U}_{v} \cap M K^{\times}\right)\left(1+\mathfrak{m}_{D, v}\right)=\mathcal{U}_{v}^{*} .
\end{gathered}
$$

We can now complete the proof of Theorem 1.2

Proof of Theorem 1.2. Let $D$ be as in Theorem 1.2, and adopt the notation of Lemma 1.3 and its proof. Let $e, f \in D$ be pure elements such that $e f=-f e$. We claim that

$$
[Y(e), X(e)]^{*}=1^{*}=[Y(e), Y(f)]^{*} .
$$

Recall that since $D$ is a quaternion division algebra, $\mathrm{SL}_{1}(D)=\left[D^{\times}, D^{\times}\right]$. Furthermore, for any valuation $v$ of a finite-dimensional division algebra, the value group $\Gamma_{v}$ is commutative. Thus $\mathrm{SL}_{1}(D)=\left[D^{\times}, D^{\times}\right] \leq \mathcal{U}_{v}$, since $\mathcal{U}_{v}$ is the kernel of the valuation $v: D^{\times} \rightarrow \Gamma_{v}$. Also the fact that the characteristic of $\bar{D}_{v}$ is two implies that $2 \in \mathfrak{m}_{D, v}$, so $2 \mathrm{SL}_{1}(D) \subseteq 2 \mathcal{U}_{v} \subseteq \mathfrak{m}_{D, v}$ and therefore

$$
S:=\left\langle 1+2 \mathrm{SL}_{1}(D)\right\rangle \leq 1+\mathfrak{m}_{D, v} .
$$

Now (iii) follows from Lemma 2.1

Let $b, c \in \mathcal{U}_{v}$ such that $[\bar{b}, \bar{c}]$ is a noncentral element in $\bar{D}_{v}\left(\bar{b}, \bar{c}\right.$ exist since $\bar{D}_{v}$ is not commutative and hence not solvable; see, e.g., [8] $)$. Let $a=[b, c]\left(=b^{-1} c^{-1} b c\right)$ and let $e \in K(a)$ be a pure element. By the choice of $a$ we have $a^{*} \neq 1^{*}$ and $a \in \mathrm{SL}_{1}(K(e)) \leq \mathcal{U}_{v}$. Thus $\left(Y(e) \cap \mathcal{U}_{v}\right)^{*} \neq 1^{*}$. Now (iii) together with Lemma 1.3 imply that $\left(\left(X(e) K^{\times}\right) \cap \mathcal{U}_{v}\right)^{*}=1^{*}=\left(\left(Y(f) K^{\times}\right) \cap \mathcal{U}_{v}\right)^{*}$. Now let $H \in\{X(e), Y(f)\}$. Then, by Lemma 1.3 (2),

$$
\left(H K^{\times} \mathcal{U}_{v}\right) /\left(H K^{\times}\left(1+\mathfrak{m}_{D, v}\right)\right) \cong \bar{D}_{v}^{\times} / \bar{K}_{v}^{\times},
$$

so $\left.D^{\times} / H K^{\times}\left(1+\mathfrak{m}_{D, v}\right)\right)$ contains a subgroup isomorphic to $\bar{D}_{v}^{\times} / \bar{K}_{v}^{\times}$. This completes the first part of Theorem 1.2 .

For the second part note first that since $\bar{D}_{v}^{\times}$is non-commutative, it contains nonabelian free groups (see, e.g., [4, Theorem 2.1]); of course, the proof of this fact uses the celebrated Tits' alternative [10. It follows that $\bar{D}_{v}^{\times} / \bar{K}_{v}^{\times}$contain a nonabelian free group. Hence $D^{\times} / X(e)$ and $D^{\times} / Y(f)$ have homomorphic images containing nonabelian free groups. But then $D^{\times} / X(e)$ and $D^{\times} / Y(e)$ also contain nonabelian free groups because if we pick one preimage for each free generator of a free subgroup in a homomorphic image, the resulting elements will generate a free subgroup.

Remarks 2.3. Note that the proof of Theorem 1.2 actually shows that if $D$ is a quaternion division algebra that supports a valuation $v$ such that the residue algebra $\bar{D}_{v}$ is non-commutative of characteristic two, and if $e, f \in D$ are pure elements such 
that $e f=-f e$, then one of $D^{\times} / Y(e)$ or $D^{\times} / Y(f)$ contains a nonabelian free group (and in fact one of these groups has a homomorphic image that contains a subgroup isomorphic to $\left.\bar{D}_{v}^{\times} / \bar{K}_{v}^{\times}\right)$.

This should be compared with the fact that for any quaternion division algebra $D$ and any pure element $e \in D$, the quotient of $D^{\times}$by the normal subgroup generated by $K(e)^{\times}$is an elementary abelian 2-group (i.e. all nonidentity elements have order 2). This last fact is rather easy to prove; cf. [7].

\section{Constructing valuations on Division algebras OF NON-COMMUTATIVE RATIONAL FUNCTIONS}

In this section we construct a quaternion division algebra whose center is an extension of $\mathbb{Q}$ of transcendence degree 2 that supports a valuation $v$ such that the residue algebra $\bar{D}_{v}$ is non-commutative of characteristic two. In view of Theorem 1.2, this will complete the proof of Theorem 1.1. More generally, we give an explicit construction of valuations on finite-dimensional division algebras of noncommutative rational functions which enables us to control the structure of the residue algebra.

Let $F$ be a field and let $v: F^{\times} \rightarrow \Gamma$ be a non-archimedean valuation on $F$. Thus $v: F^{\times} \rightarrow \Gamma$ is a nontrivial homomorphism from $F^{\times}$to a totally ordered commutative group $\Gamma$ (written additively) satisfying $v(a+b) \geq \min \{v(a), v(b)\}$, for all $a, b \in F^{\times}$, with $a+b \neq 0$. Recall that if $v(a)<v(b)$, then it follows that $v(a+b)=v(a)$.

In this section we first generalize the well-known construction of a valuation on the field of rational function $F(x)$, extending $v$ (cf. [2, Section 10.1, Proposition 2]), to a construction of a valuation on the division algebra of fractions $F(x, \sigma)$, where $\sigma \in \operatorname{Aut}(F)$ is an automorphism of finite order satisfying $v(\sigma(a))=v(a)$, for all $a \in F^{\times}$. This construction has probably been well known to experts for some time; see for example [3].

Thus let $\sigma \in \operatorname{Aut}(F)$ and let $R=F[x, \sigma]$ be the associated ring of skew polynomials in $x$. We recall that $F[x, \sigma]$ consists of formal expressions $a_{0}+a_{1} x+\cdots+a_{m} x^{m}$ with $a_{i} \in F$ which are added in the obvious way and are multiplied according to the rule: if

$$
a(x)=a_{0}+a_{1} x+\cdots+a_{m} x^{m} \text { and } b(x)=b_{0}+b_{1} x+\cdots+b_{n} x^{n},
$$

then

$$
a(x) b(x)=c_{0}+c_{1} x+\cdots+c_{m+n} x^{m+n}, \quad \text { where } c_{k}=\sum_{i+j=k} a_{i} b_{j}^{\sigma^{i}} .
$$

Now suppose that $v(\sigma(a))=v(a)$ for all $a \in F$. Define a function $w: R \backslash\{0\} \rightarrow \Gamma$ as follows: given a nonzero $a(x)=a_{0}+a_{1} x+\cdots+a_{m} x^{m}$, we let

$$
w(a(x))=\min _{a_{i} \neq 0} v\left(a_{i}\right) .
$$

Lemma 3.1. $w$ is a valuation of $R$. In other words, for nonzero $a(x), b(x) \in R$ we have

(1) $w(a(x) b(x))=w(a(x))+w(b(x))$, and

(2) $w(a(x)+b(x)) \geqslant \min \{w(a(x)), w(b(x))\}$ if $b(x) \neq-a(x)$. 
Proof. The proof of (1) is identical to the usual proof of Gauss' Lemma. Namely, suppose $w(a(x))=\alpha, w(b(x))=\beta$, and set

$$
i_{0}=\min \left\{i \mid a_{i} \neq 0, v\left(a_{i}\right)=\alpha\right\} \text { and } j_{0}=\min \left\{j \mid b_{j} \neq 0, v\left(b_{j}\right)=\beta\right\} .
$$

Then the coefficient $c_{i_{0}+j_{0}}$ of $x^{i_{0}+j_{0}}$ in $c(x)=a(x) b(x)$ is

$$
c_{i_{0}+j_{0}}=\sum_{i+j=i_{0}+j_{0}} a_{i} b_{j}^{\sigma^{i}} .
$$

If $i+j=i_{0}+j_{0},(i, j) \neq\left(i_{0}, j_{0}\right)$ and $a_{i}, b_{j} \neq 0$, we have either $i<i_{0}$ or $j<j_{0}$ and then respectively either $v\left(a_{i}\right)>\alpha$ (and $v\left(b_{j}\right) \geqslant \beta$ ) or $v\left(b_{j}\right)>\beta$ (and $v\left(a_{i}\right) \geqslant \alpha$ ). In all cases,

It follows that

$$
v\left(a_{i} b_{j}^{\sigma^{i}}\right)=v\left(a_{i}\right)+v\left(b_{j}\right)>\alpha+\beta .
$$

$$
v\left(c_{i_{0}+j_{0}}\right)=v\left(a_{i_{0}} b_{j_{0}}^{\sigma_{0}}\right)=\alpha+\beta .
$$

On the other hand, for any $k$ we have

$$
v\left(c_{k}\right)=v\left(\sum_{i+j=k} a_{i} b_{j}^{\sigma^{i}}\right) \geqslant \min _{a_{i} \neq 0 \neq b_{j}} v\left(a_{i} b_{j}^{\sigma^{i}}\right) \geqslant \alpha+\beta,
$$

and (1) follows. Property (2) is obvious.

From now on, we will assume that $\sigma$ has finite order $d$. Then the center of $R$ is $R_{0}=F^{\sigma}\left[x^{d}\right]$, where $F^{\sigma}$ is the fixed subfield. Let $S=R_{0} \backslash\{0\}$. Then $S$ is a central multiplicative subset of $R$, so the localization $D:=R_{S}$ exists, and every element of $D$ has a presentation of the form $a s^{-1}$, where $a \in R$ and $s \in S$. Note that the localization $K:=\left(R_{0}\right)_{S}$ is simply the field of fractions of $R_{0}$, and $D$ is a finite-dimensional algebra over $K$ without zero divisors, hence a division algebra. In fact, $\operatorname{dim}_{K} D=d^{2}$, as the elements $a_{i} x^{j}, i, j=1, \ldots, d$, where $a_{1}, \ldots, a_{d}$ is a basis of $F$ over $F^{\sigma}$, form a basis of $D$ over $K$. We will denote $D$ by $F(x, \sigma)$. If $a s^{-1}=b t^{-1}$, then $a t=b s$, and using Lemma 3.1(1) we immediately obtain that

$$
w(a)-w(s)=w(b)-w(t),
$$

so the equation

$$
\tilde{w}\left(a s^{-1}\right)=w(a)-w(s)
$$

yields a well-defined function on $D^{\times}$.

Lemma 3.2. $\tilde{w}$ is a valuation on $D$.

Proof. The property that $\tilde{w}(\tilde{a} \tilde{b})=\tilde{w}(\tilde{a})+\tilde{w}(\tilde{b})$ for all nonzero $\tilde{a}, \tilde{b} \in D$ immediately follows from the definition. Now, suppose we have $\tilde{a}=a s^{-1}, \tilde{b}=b t^{-1} \in D^{\times}$such that $\tilde{b} \neq-\tilde{a}$ (i.e. $b s \neq-a t$ ). By taking a common denominator we may assume that $t=s$. Suppose in addition that $\tilde{w}(\tilde{a}) \leqslant \tilde{w}(\tilde{b})$. Then

$$
\tilde{w}(\tilde{a}+\tilde{b})=w(a+b)-w(s) \geq w(a)-w(s)=\tilde{w}(\tilde{a}),
$$

and the property $\tilde{w}(\tilde{a}+\tilde{b}) \geqslant \min \{\tilde{w}(\tilde{a}), \tilde{w}(\tilde{b})\}$ follows.

Let $\mathcal{O}_{F, v}=\left\{a \in F^{\times} \mid v(a) \geq 0\right\} \cup\{0\}$ and $\mathfrak{m}_{F, v}=\left\{a \in F^{\times} \mid v(a)>0\right\} \cup\{0\}$ be the valuation ring and the valuation ideal of $v$. Note that

$$
\sigma \text { induces an automorphism } \bar{\sigma} \text { on } \bar{F}_{v} \text {, }
$$

because $\sigma$ preserves $v$. 
Lemma 3.3. Let $S_{0}=\{s \in S \mid w(s)=0\}$. Then the valuation ring and the valuation ideal of $\tilde{w}$ are as follows:

$$
\mathcal{O}_{D, \tilde{w}}=\mathcal{O}_{F, v}[x, \sigma] S_{0}^{-1} \text { and } \mathfrak{m}_{D, \tilde{w}}=\mathfrak{m}_{F, v}[x, \sigma] S_{0}^{-1} .
$$

Furthermore, the residue algebra $\bar{D}_{\tilde{w}}=\mathcal{O}_{D, \tilde{w}} / \mathfrak{m}_{D, \tilde{w}}$ is isomorphic to $\bar{F}_{v}(x, \bar{\sigma})$.

Proof. Clearly, $S=\left(F^{\sigma}\right)^{\times} S_{0}$, from which it follows that any element in $\tilde{a} \in D^{\times}$ has a presentation of the form $\tilde{a}=a s^{-1}$ with $s \in S_{0}$. Then $\tilde{w}(\tilde{a})=w(a)$, and the descriptions in (iv) easily follow. Reducing the coefficients of polynomials in $\mathcal{O}_{F, v}[x]$ modulo $\mathfrak{m}_{F, v}$ defines a surjective ring homomorphism $\varphi: \mathcal{O}_{F, v}[x, \sigma] \rightarrow \bar{F}_{v}[x, \bar{\sigma}]$ with $\operatorname{ker} \varphi=\mathfrak{m}_{F, v}[x, \sigma]$. Then $\varphi$ uniquely extends to a homomorphism of localizations

$$
\tilde{\varphi}: \mathcal{O}_{F, v}[x, \sigma] S_{0}^{-1} \rightarrow \bar{F}_{v}[x, \bar{\sigma}]\left(\varphi\left(S_{0}\right)\right)^{-1}
$$

with ker $\tilde{\varphi}=\mathfrak{m}_{F, v}[x, \sigma] S_{0}^{-1}$. It follows that $\bar{D}_{\tilde{w}} \simeq \bar{F}_{v}[x, \bar{\sigma}]\left(\varphi\left(S_{0}\right)\right)^{-1}$. As the lefthand side is a division ring, so is the right-hand side, from which it follows that it in fact coincides with $\bar{F}_{v}(x, \bar{\sigma})$ (although in general $\varphi\left(S_{0}\right)$ may be smaller than the set of nonzero elements of the center of $\left.\bar{F}_{v}[x, \bar{\sigma}]\right)$.

Now let $k$ be a field and let $u$ be a non-archimedean valuation on $k$. We use the above construction to construct a quaternion division algebra $D$ equipped with a valuation $\tilde{w}$ extending $u$ such that $\bar{D}_{\tilde{w}}$ is not commutative and has characteristic 2 .

Consider the field of rational functions $F:=k(y)$ in the variable $y$. Extend $u$ to a valuation of $F$ as above, by taking in the construction above $k$ in place of $F, u$ in place of $v$, and the identity map of $k$ in place of $\sigma$. We thus obtain a valuation $v$ on $F$ extending $u$, and

$$
\bar{F}_{v}=\bar{k}_{u}(y) .
$$

Now let $\sigma \in \operatorname{Aut}(F)$ be the unique automorphism (of order 2) taking $y \rightarrow \frac{1}{y}$ and fixing $k$ pointwise. Using the definition of $v$ one easily checks that $v(\sigma(r(y)))=$ $v(r(y))$, for any rational function $r(y) \in k(y)$. Let $D=F(x, \sigma)$ be as above. Since the order of $\sigma$ is $2, D$ is a quaternion division algebra. Also, $\bar{\sigma}$ is the unique map on $\bar{F}_{v}$ fixing $\bar{k}_{u}$ pointwise and taking $y$ to $\frac{1}{y}$. Thus $\bar{\sigma}$ is nontrivial, and since $\bar{D}_{\tilde{w}} \cong \bar{F}_{v}(x, \bar{\sigma})$, it follows that $\bar{D}_{\tilde{w}}$ is not commutative. Thus, we have shown

Proposition 3.4. Let $k$ be a field that supports a non-archimedean valuation $u$. Then there exists a quaternion division algebra $D$ whose center has transcendence degree 2 over $k$, and a valuation $v$ on $D$ extending $u$, such that the residue division algebra $\bar{D}_{v}$ is not commutative (and has characteristic equal to that of the residue field $\bar{k}_{u}$ ).

Taking in Proposition $3.4 k=\mathbb{Q}$ and $u$ the 2-adic valuation, we get the following corollary which in conjunction with Theorem 1.2 completes the proof of Theorem 1.1 .

Corollary 3.5. There exists a quaternion division algebra $D$ of characteristic zero and a valuation $v$ on $D$ such that the residue division algebra $\bar{D}_{v}$ is not commutative and has characteristic two.

\section{References}

[1] M. Aschbacher, Finite group theory, Cambridge University Press, 1986. MR0895134 (89b:20001)

[2] N. Bourbaki, Commutative algebra, chapters 1-7, translated from French. Herman, Paris; Addison-Wesley Publishing Co., 1972. MR0360549 (50:12997) 
[3] P. M. Cohn, Skew fields. Theory of general division rings, Encyclopedia of Mathematics and its Applications, 57, Cambridge University Press, Cambridge, 1995. MR.1349108(97d:12003)

[4] J. Z. Goncalves, Free groups in subnormal subgroups and residual nilpotence of the group of units of group rings, Canad. Math. Bull. 27(1984), no. 3, 365-370. MR0749646 (85k:20022)

[5] G. Prasad, The Kneser-Tits problem for triality forms, preprint, 2006.

[6] A. S. Rapinchuk, Y. Segev, and G. Seitz, Finite quotients of the multiplicative group of a finite dimensional division algebra are solvable, J. Amer. Math. Soc. 15(2002), no. 4, 929-978. MR 1915823 (2003k:16031)

[7] L. Rowen and Y. Segev, Normal subgroups generated by a single pure element in quaternion algebras, to appear in J. Algebra.

[8] W. R. Scott, On the multiplicative group of a division ring, Proc. Amer. Math. Soc. 8(1957), 303-305. MR0083984 (18:788g)

[9] Y. Segev, Pure quaternions, ultraproducts and valuations, Oberwolfach report 12/2005, 9029-9031.

[10] J. Tits, Free subgroups in linear groups, J. Algebra 20(1972), 250-270. MR0286898 $(44: 4105)$

[11] J. Tits, Groupes de Whitehead de groupes algébriques simples sur un corps (d'aprés V. P. Platonov at al.), Séminaire Bourbaki, 29e année (1976/7), Exp. No. 505, pp. 218236. MR0521771 (80d:12008)

Department of Mathematics, University of Virginia, Charlottesville, Virginia 22904

E-mail address: asr3x@unix.mail.virginia.edu

Department of Mathematics, Bar-Ilan University, Ramat Gan, Israel

E-mail address: rowen@macs.biu.ac.il

Department of Mathematics, Ben-Gurion University, Beer-Sheva 84105, Israel

E-mail address: yoavs@math.bgu.ac.il 